\title{
Entwicklung der rhetorischen Kompetenz durch Ausspracheschulung im DaF-Unterricht
}

\section{Kerstin Reinke}

Hauptziel des Fremdsprachenunterrichts ist laut Gemeinsamen europäischen Referenzrahmen (2001) die Vermittlung einer kommunikativen Sprachkompetenz, d. h. Lernende sollen auch in der Fremdsprache alle privaten und offiziellen Kommunikationssituationen angemessen sowie intentions- und wirkungsgemäß bewältigen können. Für den mündlichen Sprachbereich bedarf es daher auch einer rhetorischen Kompetenz. Ein angemessener Gebrauch phonetischer Mittel ist von hoher Relevanz, da diese in hohem Maße an der rhetorischen Wirkung beteiligt sind. Das erfordert eine gezielte Arbeit an der Aussprache, die fest in den DaF-Unterricht integriert sein muss.

Aussprache - Phonetik - Rhetorik - rhetorische Kompetenz - kommunikative Kompetenz

According to the Common European Framework of Reference, the main goal of foreign language teaching is to acquire communicative competence. Students of foreign languages should be able to master all official and private communicative situations in their foreign language. In using the foreign language, students should be able to convey their message in an effective way and achieve an impact congruent to their own intention. This means, they have to acquire (inter alia) rhetorical competence in oral language use. The rhetorical effect achieved in oral communication is significantly dependent on the appropriate use of the phonetic means available in the respective language. Gaining control of these means requires systematic training in pronunciation, which therefore should be an integral part of teaching German as a foreign language.

pronunciation - phonetics - rhetoric - rhetorical competence - communicative competence

\section{Einführung}

Der Stellenwert der Ausspracheschulung in DaF (= Deutsch als Fremdsprache) hat sich in den letzten Jahren deutlich verbessert. Neue Lehrwerke verzichten nicht mehr auf Ausspracheübungen, es erschienen einige spezielle Lernmaterialien zur Ausspracheschulung, das Interesse an Lehrerfortbildungen auf diesem Gebiet ist enorm angestiegen und nicht zuletzt verdeutlicht der GeR (= Gemeinsamer europäischer Referenzrahmen 2001), dass kommunikative Sprachkompetenz als Hauptziel des Fremdsprachenunterrichts auch Aussprachekompetenz mit einschließt.

Das bisher Erreichte darf jedoch nicht darüber hinweg täuschen, dass das Optimum - eine fest in den DaF-Unterricht integrierte Ausspracheschulung noch längst nicht erreicht ist. Immer noch wird die Aussprachekompetenz relativ losgelöst von anderen (Teil-)Kompetenzen betrachtet. Selbstverständlich erfordert Ausspracheschulung zusätzlich den Einsatz spezieller Methoden sowie einen dafür eigens eingeplanten Zeitrahmen. Zusätzlich muss sie jedoch mit allen anderen im Fremdsprachenunterricht zu vermittelnden Teilkompetenzen verknüpft werden. 
Nachfolgend soll der zentrale Stellenwert der Ausspracheschulung im Rahmen des DaF-Unterrichts herausgestellt werden. Dabei wird gezeigt, dass Ausspracheschulung auch der Vermittlung einer rhetorischen Kompetenz dient, welche wiederum mit dem Gesamtziel des Sprachunterrichts eng vernetzt bzw. sogar weitgehend identisch ist.

\section{Kommunikative Kompetenz, rhetorische Kompetenz und Ausspracheschulung in $\mathrm{DaF}$}

Zunächst soll verdeutlicht werden, inwiefern das vom GeR 2001 genannte Hauptziel des Fremdsprachenunterrichts (Erreichen einer kommunikativen Sprachkompetenz) eine Schulung der rhetorischen Kompetenz mit einschließt und welche Konsequenzen sich daraus für den Fremdsprachenunterricht und speziell für den Ausspracheunterricht in DaF ergeben.

Bei rhetorischer Kompetenz handelt es sich im Zusammenhang mit DaF weder um das Erlangen einer rhetorischen Kunstfertigkeit (Redekunst) noch um die ausschließlich wissenschaftliche Beschäftigung mit dem Gegenstand der Rhetorik. Vielmehr soll Rhetorik im Sinne von angewandter Rhetorik verstanden werden (vgl. Ueding 2006: 15), d. h. als Theorie und Praxis menschlicher Beredsamkeit in öffentlichen und privaten Angelegenheiten, die sich mit der Ausbildung, Übung und Vervollkommnung wirkungsorientierten Sprechens befasst und dazu das historisch entstandene System der Regeln, Anleitungen und Gewohnheiten benutzt.

Besonderes Augenmerk wird in der angewandten Rhetorik auf die Aspekte Wirkungsorientiertheit und Angemessenheit gelegt. Damit lassen sich Parallelen zum Hauptziel des Fremdsprachenunterrichts, der Vermittlung einer (mündlichen und schriftlichen) kommunikativen Sprachkompetenz, ziehen. Hierfür müssen linguistische, soziolinguistische und pragmatische Basiskompetenzen im Zusammenhang mit dem angemessenen (situations- und partnerorientierten) Einsatz sprachlicher Mittel entwickelt werden (vgl. GeR 2001: $21 \mathrm{ff}$.). Vor allem die mündliche kommunikative Sprachkompetenz schließt somit aufgrund ihrer Orientierung auf Angemessenheit und Wirkung sowohl in der öffentlichen (oft formellen) als auch in der privaten (informellen) Kommunikation rhetorische Kompetenz mit ein.

Unter rhetorischen Wirkungen sollen mit Verweis auf Neuber (2006: 151) „Kommunikationseffekte [verstanden werden], die auf Meinungswechsel, Einstellungswechsel und/oder Handlungsinitiierung und -wechsel hinauslaufen. "1 Auch hier sind Parallelen zu den Forderungen des GeR zu erkennen, denn der handlungsorientierte Ansatz des GeR versteht bspw. „(Kommunikative) Aufgabe[n] [...] als jede zielgerichtete Handlung, die eine Person für notwendig hält, um ein bestimmtes Ergebnis zu erzielen" (GeR 2001: 22).

1 Neuber bezeichnet das Potenzial sprachlicher - auch phonetischer - Mittel zu rhetorischen Wirkungen als „Rhetorizität“. 
Die Aussprachekompetenz wird als phonologische Kompetenz im GeR lediglich der linguistischen Basiskompetenz (GeR 2001: 117) zugeordnet. Sie hat aber auch wesentlichen Anteil an den beiden anderen Basiskompetenzen, d. h. an der soziolinguistischen und an der pragmatischen Kompetenz. Die vom GeR dort genannten Anforderungen und Ziele sind augenscheinlich ohne Einbeziehung phonetischer Mittel nicht zu bewältigen oder zu erreichen, auch wenn der GeR darauf nicht explizit eingeht. So verlangt zum Beispiel das Einhalten von Höflichkeitskonventionen in offiziellen oder privaten Gesprächssituationen (vgl. GeR 2001: 119) nicht nur die Kenntnis entsprechender syntaktischer Strukturen und Redemittel, sondern auch das Beherrschen angemessener phonetischer Mittel und insbesondere prosodischer Strukturen. Höflichkeit kann u. a. durch eine stark ansteigende Melodie am Äußerungsende angezeigt werden. Fehler auf dieser Ebene führen zu nicht intendierten Wirkungen und können negative Konsequenzen nach sich ziehen.

\section{Die rhetorische Wirkung phonetischer Mittel}

\subsection{Phonetische Mittel}

Phonetische Mittel sind artikulatorisch realisierte, auditiv wahrnehmbare und akustisch messbare Signale und Signalfolgen, die in der Wissenschaft (Phonetik) zu Beschreibungszwecken in Segmentalia (einzelne Sprachlaute) und Suprasegmentalia (den Sprachlauten aufgelagerte bzw. mehrere Sprachlaute übergreifende Phänomene wie Variationen der Tonhöhe, Dauer, Tempo, Lautheit, Spannungsgrad) unterteilt werden. In der kommunikativen Realität sind sie jedoch untrennbar miteinander verbunden.

Für den Begriff Suprasegmentalia wird in der wissenschaftlichen Literatur oft synonym der Begriff Prosodie verwendet, wobei hinsichtlich der Terminologie keine einhellige Meinung vorherrscht (vgl. Hirschfeld und Neuber 2010a: 10ff.). In einer für den DaF-Bereich praktikablen Definition wird „Prosodie als multiparametrischer Merkmalskomplex [gesehen und] umfasst Sprechmelodie, Lautheit, Dauer, Sprechgeschwindigkeit, Sprechspannung, Pausen sowie Stimmqualität/Stimmausdruck und deren jeweilige Variation. Diese Merkmale übernehmen einzeln oder in Kombination (als Akzentuierung, Gliederungssignale, rhythmische Muster) bestimmte Funktionen in gesprochenen Äußerungen, wie z. B. hervorzuheben oder zu strukturieren“ (Neuber 2010: 10f.).

Besonders beachtenswert ist, dass diese Definition sowohl stimmliche Parameter als auch das Komplexphänomen Rhythmus mit einbezieht. Denn vor allem der Sprechrhythmus hat nachweislich großen Anteil an (rhetorischen) Wirkungen. Dies wird in wissenschaftlichen sprachkontrastiven Untersuchungen immer wieder thematisiert. Auch stimmliche Parameter sind von Bedeutung, wurden jedoch bisher noch wenig untersucht (vgl. Neuber 2006). 


\subsection{Zur universellen und/oder sprachabhängigen Funktion und Wirkung phonetischer Mittel}

Phonetische Mittel als sprachliche Zeichen existieren generell in jeder Sprache und dienen universell zwei hauptsächlichen Zielen: Sie formen und transportieren das sprachliche verbale Material über entsprechende akustische Signale und Signalfolgen und sie werden gleichzeitig als Ausdrucksmittel genutzt - vgl. hierzu das Bühlersche Organonmodell (Bühler 1934), das dem sprachlichen Zeichen jeweils drei Funktionen zuweist: eine abbildende Funktion, eine Appellfunktion und eine Ausdrucksfunktion.

Die sprachliche Nachricht wird auch hinsichtlich der phonetischen Mittel universell multidimensional sowohl intendiert als auch interpretiert. Schulz von Thun (1981 und 2010: 25ff.) verdeutlicht mit seinem sogenannten 4-Seiten-Modell in Anlehnung an das Bühlersche Organonmodell, dass jede kommunizierte Nachricht hinsichtlich von vier verschiedenen Ebenen sowohl seitens der Sprecherperson intendiert als auch von den Hörern interpretiert werden kann. Intention und Interpretation können, müssen aber dabei nicht konform sein. Intentional bzw. interpretatorisch kann jeweils eine der folgenden Ebenen in den Vordergrund rücken (in Klammern jeweils Möglichkeiten der hörerseitigen Interpretation auf der genannten Ebene): a) die inhaltliche Ebene (Was wird gesagt?), b) die Ebene der Selbstdarstellung (Was ist das für ein Mensch, der mit mir spricht?), c) die Appellebene (Was soll ich denken, tun, fühlen aufgrund der Nachricht?), d) die Beziehungsebene (Wie steht die Sprecherperson zu mir?).

In der Praxis bedeutet dies, dass Sprecher- bzw. Hörerpersonen auch aus dem Repertoire der phonetischen Mittel jene einsetzen bzw. besonders beachten, die ihnen relevant erscheinen. So kann die Äußerung Es ist gleich um Fünf in Abhängigkeit der dabei verwendeten phonetischen Mittel verstanden werden als: a) Information auf der Inhaltsebene (in sachlich-neutraler Sprechweise), b) besondere Höflichkeitsäußerung auf der Selbstdarstellungsebene (in höflicher Sprechweise mit entsprechendem Tonhöhenverlauf), c) Aufforderung auf der Appellebene (in appellierender Sprechweise mit z. B. auffälligen Akzentuierungen), d) Vorwurf auf der Beziehungsebene (in vorwurfsvoller Sprechweise).

Phonetische Mittel sind generell multifunktionell. Mit besonderem Fokus auf die prosodischen Mittel, die immer auch segmentale Phänomene mit betreffen, können folgende Funktionen der Prosodie genannt werden (vgl. Miosga 2010: 56ff.; Hirschfeld und Neuber 2010: 13f.):

Linguistische (phonologische) und strukturierende Funktio n, d. h. sie geben dem Gesagten Struktur und transportieren den Inhalt. Hierzu gehört vor allem das Einhalten phonologischer Regeln, der Wortakzentregeln sowie die Gliederung von Äußerungen in rhythmische Einheiten.

Selbstexpressive und affektive Funktion, d. h. mit ihrer Hilfe kann die Sprecherperson etwas über sich selbst ausdrücken (zur emotionalen 
Befindlichkeit, über ihre Einstellung zur Mitteilung, zur Situation, zum Gesprächspartner, zur sozialen Rolle etc.).

Impressiv strukturierende Funktion, d. h. hierdurch werden innere kognitive Prozesse, wie z. B. die Abläufe der Sprechdenkvorgänge angezeigt. Diese gehen wiederum einher mit der Portionierung entsprechender Sinneinheiten, die beim Hören aufgenommen werden und auch die Aufmerksamkeit steuern. Dafür eingesetzt werden bspw. temporale Parameter, Pausen (auch gefüllte Pausen), Lautdauern und Sprechunflüssigkeiten.

Pragmatische Funktion, d. h. sie dienen zudem der Redeorganisation und wirken z. B. diskurssteuernd (Organisation des Sprecherwechsels etc.).

Die genannten Funktionen lassen sich in der Praxis nicht einfach trennen. Das zeigt sich daran, dass ein phonetisches (prosodisches) Mittel verschiedene Funktionen haben kann. Eine schwebende Melodie in der Äußerung Sie kommt nicht könnte sowohl auf Nichtabgeschlossenheit (linguistische Funktion) als auch auf Unsicherheit (selbstexpressive Funktion) hindeuten. Umgekehrt kann die gleiche Funktion durch verschiedene prosodische Mittel erfüllt werden. So ließe sich Höflichkeit in dem Satz Würden Sie bitte mal das Fenster schließen? einmal durch einen klaren Melodieanstieg am Satzende und ein andermal durch große Tonhöhenvariation innerhalb des Satzes plus fallende Melodie am Satzende ausdrücken.

Auch die phonetischen Mittel können im Hinblick auf ihre rhetorische Wirkung nur in Bezug zum komplexen Bedingungsgefüge der Kommunikation beschrieben werden. Dieses Bedingungsgefüge lässt sich durch das sogenannte Situationsmodell von Geißner (1988: 72) verdeutlichen. In diesem seit langem für die angewandte Rhetorik praktikablen Modell kommt zum Ausdruck, dass jede Situation hinsichtlich des Zusammenspiels mehrerer Variablen zu interpretieren bzw. zu beschreiben ist. Alle Variablen beeinflussen immer auch die Wahl und die Ausprägung der phonetischen Mittel. Beachtet werden sollten folgende Variablen: Wer spricht mit wem, wann, wo, warum, worüber, wie? Sobald eine Variable geändert wird, ändert sich auch die Form bzw. die Wirkung der phonetischen Mittel. Dadurch ergibt sich eine Vielzahl an beschreibbaren Mustern. Demonstriert sei dies ansatzweise mit Fokus auf die Aussprache an den folgenden Gesprächsvarianten, in denen die einzige Konstante eine Biologielehrerin an einem Gymnasium ist. Variante 1 ist eher der offiziellen Kommunikation (mit einer sachlich-neutralen Sprechweise), Variante 2 eher der inoffiziellen Kommunikation (mit einer emotional-fröhlichen Sprechweise) zuzuordnen. Unterscheidende phonetische Merkmale sind u. a. Anzahl und Ausprägung koartikulatorischer Erscheinungen (Assimilationen, Reduktionen) und Sprechtempo. 


\begin{tabular}{|c|c|c|}
\hline & Gesprächsvariante 1 & Gesprächsvariante 2 \\
\hline $\begin{array}{l}\text { Wer spricht } \\
\text { mit wem? }\end{array}$ & $\begin{array}{l}\text { Biologielehrerin } \rightarrow \text { Schüler einer } \\
\text { Abiturklasse }\end{array}$ & Biologielehrerin $\rightarrow$ eine Kollegin \\
\hline Wann? & $\begin{array}{l}\text { In einer Unterrichtsstunde zur } \\
\text { Biologie }\end{array}$ & $\begin{array}{l}\text { In der Pause, zwischen zwei } \\
\text { Unterrichtsstunden }\end{array}$ \\
\hline Wo? & Im Klassenzimmer & Im Lehrerzimmer \\
\hline $\begin{array}{l}\text { Warum } \\
\text { Worüber? }\end{array}$ & $\begin{array}{l}\text { Die Lehrerin möchte die Schüler auf } \\
\text { die Abiturprüfung vorbereiten; sie } \\
\text { beantwortet Fragen der Schüler }\end{array}$ & $\begin{array}{l}\text { Die Lehrerin möchte der Kollegin ihre } \\
\text { neuen Schuhe zeigen, weil sie sehr stolz } \\
\text { darauf ist. }\end{array}$ \\
\hline $\begin{array}{l}\text { Wie? } \\
\text { (Aussprache) }\end{array}$ & $\begin{array}{l}\text { * geringes Sprechtempo, } \\
\text { relativ laut, hoher Artikulations- } \\
\text { spannungsgrad, wenige } \\
\text { Reduktionen/Assimilationen, ... } \\
\\
\text { * phonetische Parameter der } \\
\text { sachlich-neutralen Sprechweise ... }\end{array}$ & $\begin{array}{l}\text { * hohes Sprechtempo, geringe } \\
\text { Lautstärke, hoher Artikulations- } \\
\text { spannungsgrad, viele Reduktionen und } \\
\text { Assimilationen, ... } \\
\text { * phonetische Parameter der emotionalen } \\
\text { (fröhlichen) Sprechweise ... }\end{array}$ \\
\hline
\end{tabular}

Abb. 1: Gesprächsvarianten in Bezug auf das Situationsmodell von Geißner (1988: 72)

Es kann festgestellt werden, dass sich bereits innerhalb einer Sprachgemeinschaft nur schwer eindeutige Regeln aufstellen lassen. Noch viel problematischer sind kontrastive Vergleiche zwischen verschiedenen Sprachgemeinschaften (und Sprachen) hinsichtlich der in der rhetorischen Kommunikation verwendeten phonetischen Mittel. Daher ist die Zahl der Forschungsarbeiten auf diesem Gebiet derzeit noch recht gering. Nachfolgend werden einige wichtige Aspekte angesprochen, die hinsichtlich solcher kontrastiver Vergleiche bedacht werden sollten.

Zunächst ist zu beachten, dass phonetische Signale als artikulatorisch erzeugte und auditiv wahrnehmbare (bzw. akustisch messbare) Signale teilweise biologisch determiniert sind. Der Artikulationstrakt (Ansatzrohr mit Artikulationsorganen und Resonanzräumen sowie das Stimmerzeugungsorgan) ist bei allen Menschen ähnlich aufgebaut, jedoch lassen individuelle Unterschiede teilweise Rückschlüsse auf Figur und Größe des Sprechers zu. Muskuläre Spannungsänderungen - wie sie beispielsweise bei emotionaler Involviertheit auftreten - wirken sich bei jedem Menschen auf die Artikulationsmuskulatur aus und prägen dadurch das phonetische Signal. Auch hier sind die Faktoren biologische Determiniertheit und Individualität wirksam (vgl. Reinke 2011: 73). Zum großen Teil ist das phonetische Signal aber auch überformt durch die vorgegebenen Normen der Sprachgemeinschaft, die soziokulturell erworben werden und die sowohl Intentionen und Interpretationen hinsichtlich der phonetischen Signale prägen. Das lässt sich z. B. beim Ausdruck sozial angemessener Emotionen wie dem Anzeigen von Freundlichkeit in bestimmten Situationen erkennen. Auch in Bezug auf offizielle Redesituationen gibt es oft recht klare Erwartungsnormen bezüglich des Einsatzes phonetischer Mittel an einen guten Redner/eine gute Rednerin (vgl. Hirschfeld und Neuber 2010b: 49ff.). 
Interferenzen auf der Ebene des Sprechrhythmus (als zur Prosodie gehörendes Komplexphänomen) sind im Vergleich zu segmentbetreffenden Interferenzen noch weniger gut erforscht. Die bisherigen Forschungsergebnisse beziehen sich hier meistens auf die sogenannte sachlich-neutrale Sprechweise (gemeint ist damit meist eine relativ emotionsfreie Sprechweise mit neutraler Artikulationsspannung), die aber in der kommunikativen Realität nur eingeschränkt angemessen ist (vgl. Reinke 2008: 258).

Mit Verweis auf Neuber (2010: 77) sollten folgende Themen auf der Ebene der Prosodie/des Sprechrhythmus aufgrund ihrer rhetorischen Wirkungsrelevanz und der Interferenzproblematik in künftigen Forschungsarbeiten und im DaF-Unterricht Beachtung finden:

Signalportionierung (Länge und Struktur zusammenhängend gesprochener Silben- oder Wortfolgen bzw. Sprechtakte) und Informationsprofil (z. B. Einsatz phonetischer Mittel, die wichtige Informationen verdeutlichen): Im Deutschen können Sprechtakte eine hohe Silbenzahl aufweisen, wobei die Energie sich besonders auf betonte Silben zentriert. Vielen Lernenden fällt es schwer, diese relativ langen Sprechtakte zusammenhängend mit dem entsprechenden Betonungsmuster zu realisieren. Werden z. B. in Vorträgen zu viele Pausen gesetzt und wird nicht angemessen betont, kann u. a. die Verständlichkeit beeinträchtigt und die Sprecherperson als weniger kompetent empfunden werden.

Phonostilistische Markierungen: Im Deutschen sind mehr oder weniger reduzierte bzw. totalassimilierte Endsilben häufig ein Indiz für den entsprechenden Offizialitätsgrad. Je informeller die Kommunikationssituation, umso mehr totalassimilierte Endungen sind anzutreffen. In vielen Sprachen sind derartige koartikulatorische Prozesse nicht üblich. Lernenden mit diesen Ausgangssprachen fällt es schwer, in informellen Situationen (z. B. Smalltalk) entsprechende phonostilistische Markierungen vorzunehmen. Sie könnten aufgrund dessen als weniger sympathisch eingeschätzt werden.

Situativ-pragmatische Applikationen: Zum Beispiel wird eine freundlicheSprechweise in jeder Sprachgemeinschaft in bestimmten kommunikativen Situationen erwartet. Im Deutschen empfindet man eine freundliche Sprechweise bspw. in einem Verkaufsgespräch als angemessen. Sie zeichnet sich meist aus durch größere Tonhöhenvariationen innerhalb der Äußerung bzw. durch ansteigende Melodie am Äußerungsende. Fehlen diese Parameter, so wird die Sprecherperson ggf. als unhöflich eingeschätzt.

Dialog ste u e rung: Bestimmte prosodische Signale regulieren zum Beispiel den Sprecher- und Hörerwechsel in Gesprächen. Im Deutschen signalisiert ein Absenken der Tonhöhe bis in die Lösungstiefe am Ende von Äußerungen häufig, dass die Sprecherrolle abgegeben werden soll. Schwebende Melodie am Äußerungsende, gefolgt von einer längeren Pause wird dagegen oft als Unsicherheit aufgefasst, so dass ggf. einer Sprecherperson die Sprecherrolle-auch gegen ihren Willen-entzogen wird. 


\section{Probleme und Möglichkeiten in DaF}

Die o. g. Ausführungen haben gezeigt, dass phonetische Mittel erheblichen Anteil an rhetorischer Wirkung haben, so dass der Ausspracheunterricht zur Schulung der rhetorischen Kompetenz beitragen kann und auch muss. Allerdings sind dabei einige Aspekte zu beachten:

Noch besteht ein Defizit an wissenschaftlichen Analysen und Ergebnissen. Somit sind entsprechende Didaktisierungsvorschläge und Übungen zur Ausspracheschulung in Verbindung mit der Schulung der rhetorischen Kompetenz, von einigen Ausnahmen abgesehen (Lehrwerke: Dimensionen 2002ff. und Aussichten 2009ff., Aussprachelernmaterialien: 33 Aussprachespiele 2009 und Phonetiktrainer A 1-B 1 2012), bisher leider rar. Auch spiegelt sich die reale rhetorische Vielfalt noch zu wenig in den Höraufnahmen von Lehrwerken wider. Dort werden Alltagsgespräche oft in einer unangemessen hohen phonostilistischen Variante angeboten (vgl. Reinke 2007: $180 \mathrm{ff}$.). Es ist empfehlenswert, im Unterricht möglichst viele (quasi)-authentische Hör- und Videobeispiele einzusetzen, in denen reale Kommunikationssituationen gezeigt werden. Nur so werden Lernende für neue phonetische Muster in ihrer gesamten Variantenbreite sensibilisiert.

Dazu ein Beispiel aus dem Lehrwerk Aussichten A2, in dem ein gut inszeniertes „Alltagsgespräch“"zwischen zwei Freunden in einer Disco mit Musik im Hintergrund zu hören ist. Die Lernenden werden aufgefordert, auf spezifische phonostilistische Varianten (Elisionen) in der Alltagssprache zu achten und sie anschließend selbst produktiv - gemäß eines vorgegebenen Musters - auszuprobieren.

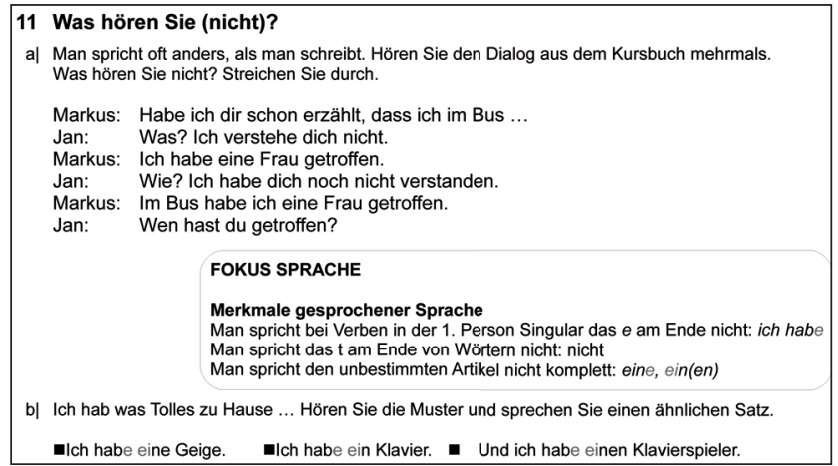

Abb. 2: Ausspracheübung zu phonostilistischen Merkmalen der Alltagssprache aus Aussichten A2, Arbeitsbuch, Lektion 11 (2011: 11)

Man sollte außerdem bedenken, dass nicht alle Lernenden gleichermaßen bereits aus ihrer Muttersprache über ausreichend rhetorische Kompetenzen (v. a. für formelle Kommunikationssituationen) verfügen bzw. in der Lage sind, darüber im Vergleich 
zu den Besonderheiten in der Fremdsprache zu reflektieren. Insofern müssen Lernende im DaF-Unterricht zur Reflexion über Intention und Wirkung sowohl für ihre eigene Muttersprache als auch für die Fremdsprache DaF sensibilisiert werden. Ausgangspunkt wird dabei oftmals die zu erlernende Fremdsprache (Deutsch) sein. Die Aufmerksamkeit der Lernenden sollte gezielt auf solche phonetischen Merkmale gelenkt werden, die eine entsprechende rhetorische Wirkung erzielen. Aus dieser Perspektive wäre dann ein Vergleich zur jeweiligen Muttersprache anzuregen.

Auch individuelle Unterschiede sind zu beachten. Das bedeutet, dass selbst innerhalb einer Sprachgemeinschaft Menschen mehr oder weniger geschickt sind, phonetische Mittel im privaten oder öffentlichen Bereich effizient einzusetzen. Zurückhaltende Lernende sollten daher besonders aufmerksam und sensibel gefördert werden.

Zu berücksichtigen ist auch, dass in der rhetorischen Kommunikation eine gewisse Toleranzbreite existiert (zur Richtigkeitsbreite vgl. Forster 2004: 121ff.). Fixe Regeln sollten daher sehr vorsichtig vermittelt werden und nur da, wo wirklich möglich und nötig. Vielmehr sollen Wahlmöglichkeiten für kommunikatives Verhalten angeboten werden, indem über Wirkung diskutiert wird mit Fragestellungen wie: Wie gut gefällt Ihnen die Sprechweise in dieser Situation? Warum (nicht)? Möchten Sie auch so sprechen? In welcher Situation (nicht)?

In der nachfolgenden Übung aus Aussichten A2 zum Gesprächsverhalten als Zuhörer (Abb. 3) geht es auch (aber nicht nur) um die Sprechweise. Die Lernenden werden aufgefordert, bestimmte Verhaltensweisen selbst auszuprobieren und für sich zu bewerten. Außerdem werden sie angeregt, andere Personen zu beobachten (im Alltag, in den Medien), um sich selbst eine Meinung bilden zu können. Ein solches Übungskonzept lässt sich nutzen, um auf verschiedene Faktoren der Sprechweise in unterschiedlichsten Situationen zu achten.

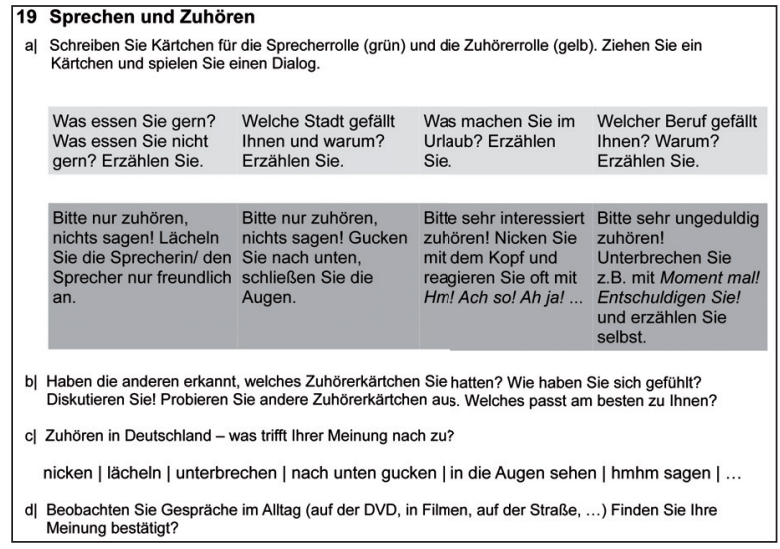

Abb. 3: Ausspracheübung zur Zuhörerrolle aus Aussichten A2, Kursbuch, Lektion 15 (2011: 86) 
In der nachfolgenden Übung (Abb. 4) aus Aussichten B 1 sollen die Lernenden verschiedene Sprechrollen ausprobieren und danach über ihre eigene Wirkung und ihre Vorstellungen über geltende Gewohnheiten in ihrer Muttersprache reflektieren sowie Beobachtungen dazu machen.

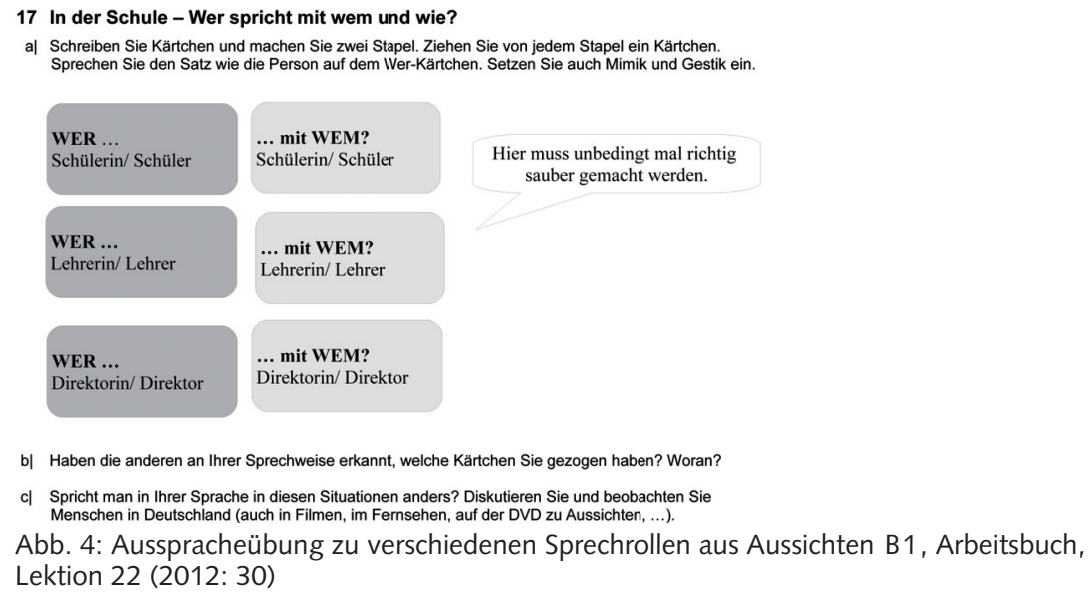

b) Haben die anderen an Ihrer Sprechweise erkannt, welche Kärtchen Sie gezogen haben? Woran?

c| Spricht man in Ihrer Sprache in diesen Situationen anders? Diskutieren Sie und beobachten Sie Menschen in Deutschland (auch in Filmen, im Fernsehen, auf der DVD zu Aussichten, ...).

Abb. 4: Ausspracheübung zu verschiedenen Sprechrollen aus Aussichten B1, Arbeitsbuch, Lektion 22 (2012: 30)

Aufgrund der Multifunktionalität phonetischer Mittel und ihrer Abhängigkeit von weiteren Variablen der Kommunikation (vgl. Situationsmodell usw.) entsteht eine große Variantenbreite mit unzähligen Wirkungsmöglichkeiten, die im DaFUnterricht niemals komplett vermittelbar ist. Hier muss - entsprechend den Erfordernissen (in Bezug auf den GeR und abhängig vom Ausbildungsziel) - eine entsprechende Auswahl getroffen werden.

Außerdem sind die verschiedenen Funktionen der phonetischen Mittel - wie oben beschrieben - miteinander sehr eng verwoben. Lernenden sollten daher Kenntnisse über diese Multifunktionalität vermittelt werden. Hinsichtlich der Funktion von steigender Melodie am Äußerungsende kann z. B. in entsprechenden kommunikativen Kontexten darauf hingewiesen werden, dass man damit sowohl eine Frage anzeigen als auch besondere Höflichkeit markieren kann.

Die Unterrichtssituation selbst bietet nur eingeschränkte Möglichkeiten, verschiedene Situationen und Rollen authentisch zu erleben, zu realisieren. Es sollten also Situationen inszeniert werden, wobei Lehrende und Lernende in unterschiedliche Rollen schlüpfen und dafür passende phonetische Mittel einsetzen können. Gut geeignet sind Sprechstücke, Theatersequenzen, Rollenspiele u. ä. Danach sollte jeweils diskutiert werden, inwieweit die Sprechweise zur Rolle und zur Situation gepasst hat und welche phonetischen Mittel die Wirkung erzeugt haben. 


\section{Fazit}

Rhetorisch kompetent zu sein heißt also, dass man vor allem mündliche kommunikative Situationen sowohl im privaten als auch im öffentlichen bzw. offiziellen Rahmen bewältigen kann. Dies ist - wie gezeigt werden sollte - auch angestrebtes Ziel des Fremdsprachenunterrichts DaF. Da phonetische Mittel einen erheblichen Anteil an rhetorischen Wirkungen haben, müssen sie im DaFUnterricht eine viel stärkere Beachtung finden. Bekräftigt werden muss hier vor allem noch einmal die Forderung, Ausspracheübungen stärker in den DaFUnterricht zu integrieren. Es genügt nicht, die Anforderungen des Gemeinsamen europäischen Referenzrahmens zu erfüllen, sondern in der Praxis muss noch weit darüber hinausgegangen werden.

\section{Literaturverzeichnis}

Bühler, Karl (1934): Grundmodell der Kommunikation. Sprachtheorie. Jena.

Forster, Roland (2004): Zur Diskussion einer Richtigkeitsbreite in der mündlichen Kommunikation. In: Altmayer, Claus / Forster, Roland / Grub, Frank Thomas (Hg.). Deutsch als Fremdsprache in Wissenschaft und Unterricht: Arbeitsfelder und Perspektiven. Festschrift für Lutz Götze zum 60. Geburtstag. Frankfurt a. M. [u. a.], Peter Lang. S. $121-130$.

Geißner, Hellmut (1988): Sprechwissenschaft. Theorie der mündlichen Kommunikation. Frankfurt a. M., Scriptor.

Hirschfeld, Ursula / Neuber, Baldur (2010a): Prosodie im Fremdsprachenunterricht Deutsch - ein Überblick über Terminologie, Merkmale und Funktionen. In: Deutsch als Fremdsprache 47/1/2010. S. 10-16.

Hirschfeld, Ursula / Neuber, Baldur (2010b): Methodische Überlegungen zur Untersuchung phonetischer und rhetorischer Parameter im interkulturellen Wissenschaftsdiskurs. In: Sprechen. Zeitschrift für Sprechwissenschaft, Sprechpädagogik, Sprechtherapie, Sprechkunst 49/2010. S. 45-55.

Hirschfeld, Ursula / Reinke, Kerstin (2009): 33 Aussprachespiele. Stuttgart, Klett.

Jenkins, Eva-Maria / Clalüna, Monika / Fischer, Roland / Hirschfeld, Ursula (2002ff.): Dimensionen. Lehrwerk Deutsch als Fremdsprache 1-3. Ismaning, Hueber.

Miosga, Christiane (2010): Der Ton macht die Musik. Terminologische Diskussion zur Prosodie und ihr Nutzen für Theorie und Praxis. In: Sprechen. Zeitschrift für Sprechwissenschaft, Sprechpädagogik, Sprechtherapie, Sprechkunst 50/2010. 48-66.

Neuber, Baldur (2006): Phonetische und rhetorische Wirkungen sprechstimmlicher Parameter. In: Deutsch als Fremdsprache 43/3/2006. S. 151-156.

Neuber, Baldur (2010): Funktionen der Prosodie im Blickwinkel der interkulturellen Kommunikation. In: Hirschfeld, Ursula / Stock, Eberhard (Hg.). Sprechwissenschaftlichphonetische Untersuchungen zur interkulturellen Kommunikation Russisch - Deutsch. Frankfurt. a. M. [u. a.], Peter Lang. S. 69-80.

Reinke, Kerstin (2007): Befähigung zu kommunikativer Kompetenz - kommunikative Realität und phonetische Varianten in DaF-Lehrwerken. Frankfurt a. M., Peter Lang.

Reinke, Kerstin (2008): Zur Wirkung phonetischer Mittel in sachlich intendierter Sprechweise bei Deutsch sprechenden Russen. Frankfurt a. M., Peter Lang. 
Reinke, Kerstin (2011): Fremder Akzent - von der akustischen Wahrnehmung zur Deutung der Persönlichkeit. In: Babylonia. Die Zeitschrift für Sprachunterricht und Sprachenlernen. 21/2/2011. S. 73-79.

Reinke, Kerstin (2012): Phonetiktrainer A1 - B1. Stuttgart, Klett.

Ros-El Hosni, Lourdes / Swerlowa, Olga / Klötzer, Sylvia / Jentges, Sabine / Sokolowski, Katrin / Reinke, Kerstin / Precht, Jörn / Lundquist-Mog, Angelika / Reicherter, Angelika (2009ff.): Aussichten. Deutsch als Fremdsprache für Erwachsene A1 - A2. Stuttgart, Klett.

Schulz von Thun, Friedemann (1981 und 2010): Miteinander reden. Störungen und Klärungen 1. Hamburg, Rowohlt.

Ueding, Gert (2006): Was ist Rhetorik? In: Soudry, Rouven (Hg.). Rhetorik: Eine interdisziplinäre Einführung in die rhetorische Praxis. Heidelberg [u. a.], C. F. Müller. S. 13-23. 